\title{
A 16-MONTH ANALYSIS OF URINARY TRACT INFECTION IN CHILDREN
}

\author{
G. R. E. NAYLOR*
}

\section{Gonville and Caius College, Cambridge CB2 ITA}

\begin{abstract}
SUMmaRY. Results of the examination of urine specimens with evidence of acute urinary tract infections from children aged 16 years and under in general practice were analysed during a period of 16 months. Infections were much commoner in girls than in boys, with Escherichia coli most frequently involved in both groups. Urinary tract infections caused by Proteus strains were predominantly associated with boys. Infections in girls showed a higher incidence at 3,6 and 16 years of age. Possible reasons for these sex- and age-associated patterns of infection are discussed. It is suggested than an important factor in the prevention of urinary tract infections in young girls is proper supervision of school lavatories. The report illustrates how much useful information can be obtained from the analysis of diagnostic results based on a simple but thorough laboratory approach.
\end{abstract}

\section{INTRODUCTION}

Infections of the urinary tract in children are particularly important because their occurrence may be associated with some congenital abnormality of the urinary tract or some error in management which, if uncorrected, may lead to recurrent infections with further damage to the urinary tract.

This report is based on investigations of urine specimens from children aged 16 years and under, submitted mainly from general practice but also from a home for mentally handicapped children. The specimens were not from hospital patients. As the general practitioners were not aware of this analysis, the specimens examined represent an unsolicited sample.

\section{MATERIALS AND METHODS}

\section{The Survey}

This was based on the diagnostic work of a clinical bacteriology laboratory serving Cambridge and district. The laboratory handled c. 80000 specimens/year. During the 16-month period of the survey from September 1974 to December 1975, the laboratory dealt with c. 104000 specimens including c. 23000 samples of urine.

Received 8 Jun. 1983; accepted 20 Jun. 1983.

* Dr Naylor died in August 1983, just after completing this paper. 
Copies of all reports on urine specimens from children aged 16 years and under were collected. Specimens showing an abnormal increase in leukocyte or red cell content and a growth of colonies of identical appearance (regarded as a pure culture) and representing $>10^{5}$ organisms $/ \mathrm{ml}$ of the original urine, were identified as indicating infection of the urinary tract. In the great majority of such specimens, leukocytes were greatly increased with no more than a few red cells present; red cells predominated in a small number of specimens and these probably represented early infections. Specimens submitted from the same child within 1-3 weeks were regarded as duplicates and only one report was retained.

Urine specimens that were not examined within $6 \mathrm{~h}$ after collection, either because of late evening surgeries or because the surgery was distant from the laboratory, were accompanied by a dip-slide and the estimate of the bacterial concentration in the fresh specimen was judged from the dip-slide instead of the semi-quantitative culture in the laboratory (Naylor and Guttmann, 1967).

\section{Laboratory techniques}

Examination of urines for cells. About $5 \mathrm{ml}$ of urine was centrifuged, the supernate tipped off and the deposit resuspended in the urine that drained back $(c .0 .1 \mathrm{ml})$. A wet film of the suspension was examined microscopically with the $\times 40$ objective and the $\times 100$ objective. More than two or three white cells per field with the $\times 100$ objective were regarded as abnormal. The presence of any red cells was regarded as abnormal.

Culture. In general, urines were cultured on a Macba plate (Naylor, 1980) which is a plate poured in two halves, of horse blood agar and MacConkey agar. The plate was sown with a measured drop of urine transversely across the two media; an additional heavily sown strip down the margin of the blood agar half accommodated four antibiotic sensitivity disks. The Macba plate yielded a semi-quantitative bacterial count and allowed observations of colonial appearances on blood agar and MacConkey agar and sensitivity determinations to the four agents (Olds, 1975). The sensitivity disks used were ampicillin $25 \mu \mathrm{g}$, nitrofurantoin $200 \mu \mathrm{g}$, nalidixic acid $30 \mu \mathrm{g}$ and sulphafurazole $200 \mu \mathrm{g}$. Organisms were reported as sensitive if the zone of inhibition of growth was $5 \mathrm{~mm}$ or more from the margin of the disk (a total inhibition zone of at least $16 \mathrm{~mm}$ in diameter). Proteus strains were classified as resistant to nitrofurantoin irrespective of the in-vitro test (Brumfitt and Percival, 1967).

Occasionally, urine was sown on separate blood agar and MacConkey agar plates and heavily on an additional blood agar plate or plates to determine further sensitivities. Urines delayed in transit were accompanied by a dip-slide to allow a valid bacterial count.

Bacterial identification was solely by examination of the overnight culture with a hand lens. This simple approach is adequate for routine examination of diagnostic specimens and treatment of most patients and is based on the following criteria. Not all the cultures were examined by the author but he and his colleagues were sufficiently familiar with each other's practice for the results to be comparable.

The simple criteria were: Escherichia coli produces large colonies, $c .3 \mathrm{~mm}$ in diameter, that ferment lactose. Coliform bacilli produce large colonies not fermenting lactose. No attempt was made to differentiate colonies with a moist or mucoid appearance. (This group is very mixed and it is conceded that it includes Salmonella typhi if a urinary carrier is encountered.) Proteus colonies show swarming on blood agar. Pseudomonas aeruginosa does not ferment lactose and is oxidase-positive. Staphylococcus albus produces opaque white colonies $c .2 \mathrm{~mm}$ in diameter on blood agar; the organism grows very poorly on MacConkey agar; it is resistant to nalidixic acid. No distinction was made between $S$. saprophyticus, S. epidermidis and Micrococcus spp. Streptococcus faecalis yields small colonies $c .1 \mathrm{~mm}$ in diameter on blood agar and smaller, just visible colonies on MacConkey agar; the growth is resistant to nalidixic acid.

A special attempt was made to detect cysteine-dependent strains of $E$. coli. These organisms produce small $(1 \mathrm{~mm})$ colonies on blood agar and MacConkey agar and are sensitive to nalidixic acid; their identity can be checked by applying a paper disk impregnated with cysteine hydrochloride to the surface of the medium and reincubating, when the colonies of cysteine-dependent strains enlarge within 4-6 h. No cysteine-dependent organisms were detected in the present series. 


\section{RESULTS AND DISCUSSION}

Urine reports collected during the continuous 16-month period totalled 2559 from 1838 patients after duplicates were discarded. Nothing abnormal was found in 1656 specimens. Evidence of bacterial infection, as defined above, was recorded in 303 reports ( 240 children). Other abnormal findings were: 73 reports recorded a pure growth of bacteria in excess of $10^{5} / \mathrm{ml}$ without increase in cell content; 384 reports recorded an increase in either white cells alone or a mixture of red cells and white cells in various proportions without significant numbers of bacteria; 52 reports recorded proteinuria without an increase in cell content or significant bacterial growth; and 25 reports recorded glycosuria. Reports on 66 specimens were impossible to classify for a variety of reasons, such as contamination, bacterial growth in a stale specimen, or mixed bacterial growth accompanied by an increase in white cells.

Analyses of infections are presented in figs. 1 and 2.

\section{Infecting organism}

E. coli was the commonest organism found in both boys and girls.

A Proteus sp. was identified as the causative organism much more frequently in boys than in girls. Perhaps this is because Proteus is a more common inhabitant of the preputial sac than the vulva; perhaps it is because Proteus, with its active motility and swarming ability can, in comparison with other organisms, more readily traverse the longer urethra in boys than the shorter urethra in girls. Maskell and Pead (1976) also noted the particular association of Proteus infections with boys.

\section{Age and sex}

Infections were very much more commonly diagnosed in girls than in boys, particularly with $E$. coli and coliforms.

In girls, the age incidence of infection with $E$. coli showed three peaks at 3,6 and 16 years. The higher incidence at 3 and 6 years may represent the beginning of nursery school and infant school when young girls face, for the first time, errors in self-management and the embarrassments of micturating away from home. Errors in management made by schoolgirls are to cleanse the perineum forwards from the anus to the vulva and to micturate infrequently. Infrequent micturition leads to overdistension of the bladder with subsequent incomplete emptying; residual urine then interferes with bladder defences by allowing bacterial colonisation of bladder urine to occur more readily. The high incidence at 16 years probably reflects the beginnings of sexual experience; indeed, a few of the 16-year-olds were pregnant (Nicolle et al., 1982).

Among the patients submitting specimens, there were some with abnormalities of the urinary tract who had recurrent infections during the 16-month period, often with different organisms. As this could influence the derived age incidence data, we sought to confirm the age distribution of infections in girls based on specimen numbers (fig. 1) by recording the age incidence of girls suffering at least one urinary tract infection in the 16-month period by patient numbers in fig. 2 . The age incidence of infections in girls again showed peaks at 3,6 and 16 years. This emphasizes the need for adequate 


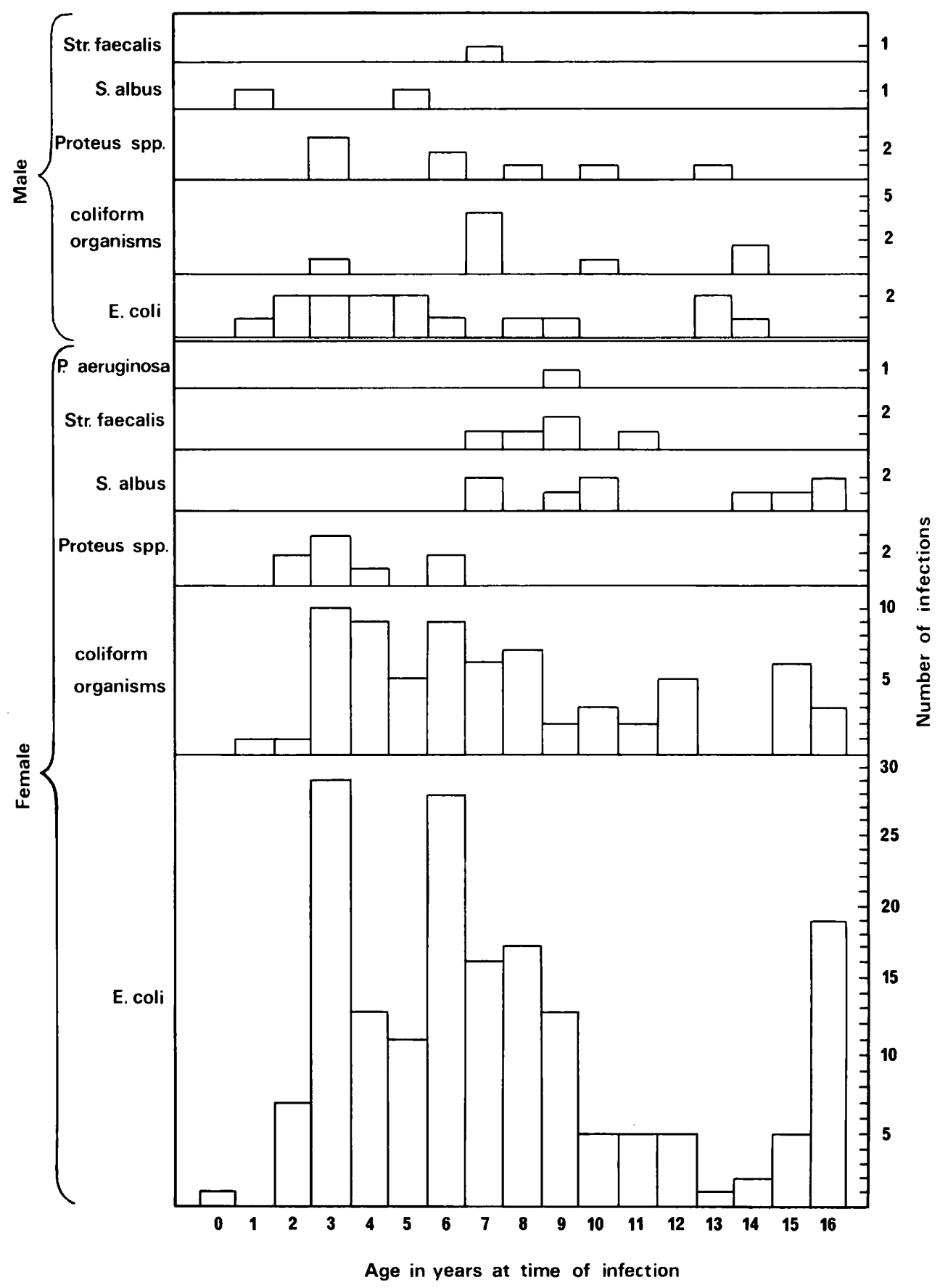

FIG. 1.--Histogram indicating organisms associated with urinary tract infections in 240 children aged 16 years or less. 


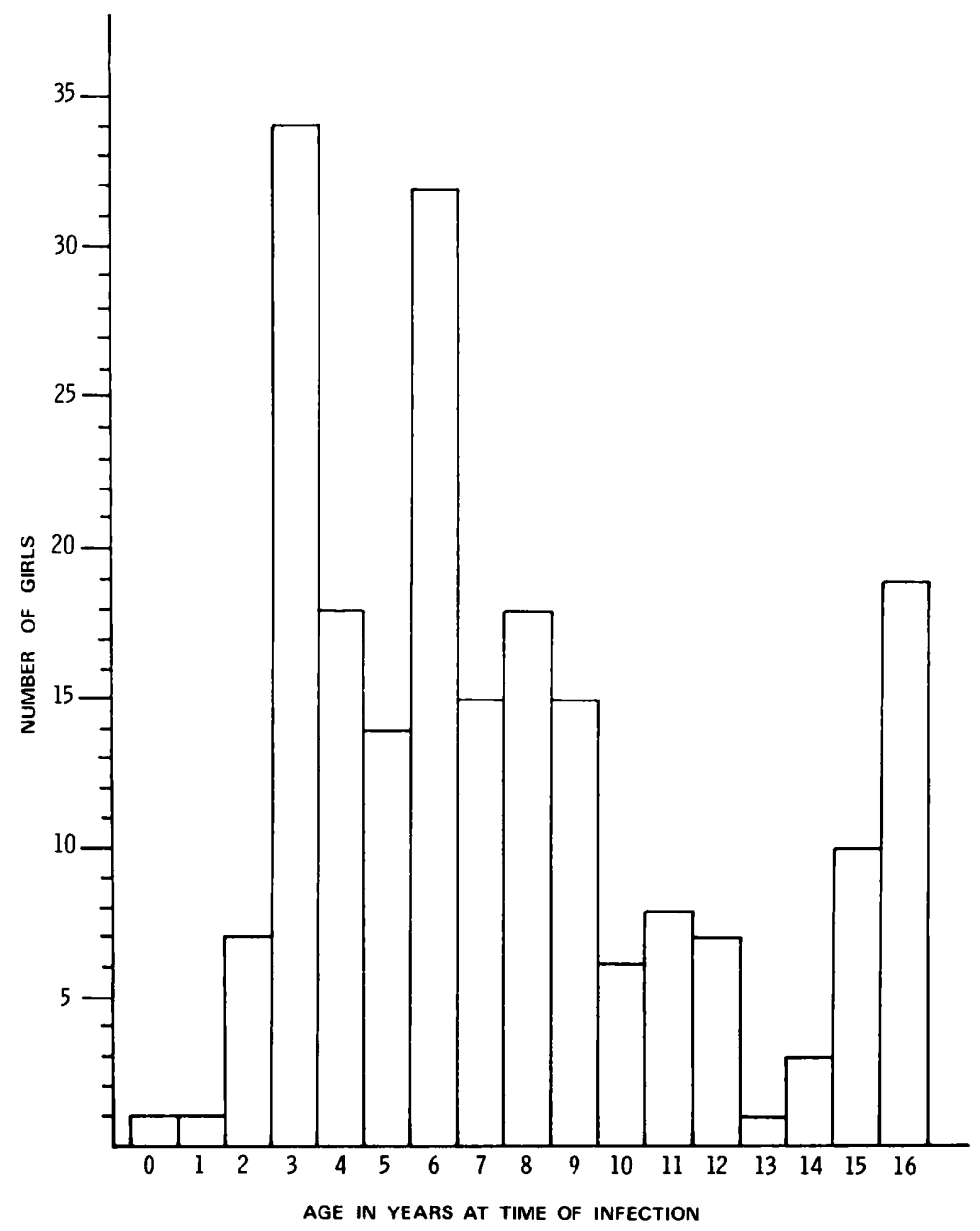

FIG. 2.-Histogram indicating numbers of female patients aged 16 years and under with at least one urinary tract infection in the 16-month study period.

and careful supervision of school lavatories so that shy young girls starting school can micturate in peace at school without disturbance, embarrassment or molestation.

The laboratory work in this analysis was very simple and more laboratory effort with modern facilities now available, particularly in the classification of Staphylococcus species and identification of the coliform group, might be helpful. On the other hand, one great advantage of the simple laboratory methods is speed, with a short time interval between receiving the specimen and issuing the report. It is always important to ensure that any delay in issuing the report caused by more thorough identification of the causative organism is worthwhile in terms of improved patient care.

It is encouraging that so much useful information, entirely consistent with results and analyses of much more sophisticated surveys, can be derived from relatively simple but thorough routine laboratory work together with the close clinical liaison on which this study is based. The suggestion for the prevention of urinary tract infections in young girls to emerge from this simple analysis can readily be put into practice by 
general medical practitioners and school medical officers advising parents and teachers.

This work was done while the author was University Director of the Public Health Laboratory Service at Cambridge. He is very grateful to his medical colleagues, Dr J. M. Boissard, Dr J. Nagington and Dr R. S. B. Wickremesinge, for their help in the examination of the urine cultures and to Mr V. C. T. Rolfe and the technical staff of the laboratory for all their work in the preparation of the culture media and the handling of the specimens. He acknowledges with much gratitude the help of Miss Patricia Rowlands in the collection of the laboratory reports, and he thanks Professor J. G. Collee for his advice and encouragement.

\section{REFERENCES}

Brumfitt W, Percival A 1967 Laboratory control of antibiotic therapy in urinary tract infection. Annals of the New York Academy of Sciences 145:329-343.

Maskell R M, Pead L J 1976 Urinary infection in children in general practice: a laboratory view. Journal of Hygiene 77:291-298.

Naylor G R E 1980 Technique for the cultural examination of urine using a single plate (the Macba plate). Journal of Hygiene 84:429-432.

Naylor G R E, Guttman D 1967 The dip-slide: a modified dip-inoculum transport medium for the laboratory diagnosis of infections of the urinary tract. Journal of Hygiene 65:367-371.

Nicolle L E, Harding G K M, Preiksaitis J, Ronald A R 1982 The association of urinary tract infection with sexual intercourse. Journal of Infectious Diseases 146:579-583.

Olds R J 1975 A colour atlas of microbiology. Wolfe Medical Publications, London, p 161. 